\title{
Insulin treatment attenuates diabetes-increased atherosclerotic intimal lesions and matrix metalloproteinase 9 expression in apolipoprotein E-deficient mice
}

\author{
Corinne A Schuyler ${ }^{1}$, Nga $\mathrm{N} \mathrm{Ta}^{2}$, Yanchun $\mathrm{Li}^{2}$, Maria F Lopes-Virella ${ }^{1,2}$ and Yan Huang ${ }^{1,2}$ \\ ${ }^{1}$ Ralph H. Johnson Veterans Affairs Medical Center, Charleston, South Carolina 29401, USA \\ ${ }^{2}$ Division of Endocrinology, Diabetes and Medical Genetics, Department of Medicine, Medical University of South Carolina, Charleston, South Carolina \\ 29425, USA \\ (Correspondence should be addressed to Y Huang who is at Division of Endocrinology, Diabetes and Medical Genetics, Department of Medicine, Medical \\ University of South Carolina, 114 Doughty Street, Charleston, South Carolina 29403, USA; Email: huangyan@musc.edu)
}

\begin{abstract}
Patients with diabetes mellitus have increased mortality and morbidity of cardiovascular diseases compared with nondiabetic patients. Although clinical studies have shown that effective glycemic control with insulin treatment in patients with type 1 diabetes is associated with reduced cardiovascular events, the underlying mechanisms have not been well understood. In this study, we treated diabetic apolipoprotein E-deficient (apoE-/-) mice with insulin for 20 weeks and studied the effect of insulin treatment on intimal lesion size and matrix metalloproteinase (MMP) 9 expression known to be involved in plaque destabilization. Results showed that insulin treatment, which effectively reduced plasma glucose
\end{abstract}

level in diabetic mice, attenuated diabetes-increased intimal lesion size and significantly inhibited diabetes-increased MMP9 expression, but had no effect on tissue inhibitor of metalloproteinase 1 in atherosclerotic plaques. Furthermore, we observed that insulin treatment did not reduce diabetes-increased macrophage content but inhibited interleukin 6 expression, a stimulator for MMP expression. Taken together, this study has shown for the first time that insulin treatment in diabetic apoE $-/-$ mice changes atherosclerotic lesions and gene expression to a state that favors plaque stability.

Journal of Endocrinology (2011) 210, 37-46

\section{Introduction}

Epidemiological studies have consistently shown that patients with diabetes mellitus have increased mortality and morbidity of cardiovascular disease compared with the general population (Soedamah-Muthu \& Stehouwer 2005). To determine whether the control of hyperglycemia, the hallmark of diabetes, reduces the risk of cardiovascular events, a clinical study entitled 'Diabetes Controls and Complication Trial/ Epidemiology of Diabetes Interventions and Complications (DCCT/EDIC)' was conducted on 1441 patients with type 1 diabetes (Nathan et al. 2005). After giving patients either conventional or intensive insulin treatment, which lowered HbAlc level to $9 \cdot 1$ or $7 \cdot 4 \%$ respectively, for 6.5 years and following up patients for 17 years, the study group reported that the intensive treatment significantly reduced the risk of any cardiovascular disease event by $42 \%(P=0.02)$ and the risk of nonfatal myocardial infarction, stroke, or death from cardiovascular disease by $57 \%(P=0 \cdot 02)$. Furthermore, the study showed that the decrease in HbAlc values was significantly associated with most of the positive effects of intensive treatment on the risk of cardiovascular disease.
Clearly, this study provided evidence to support an essential role of glycemic control in reduction in mortality and morbidity of cardiovascular disease in patients with type 1 diabetes. However, it remains unclear how glycemic control decreases cardiovascular events and, in particular, the effect of glycemic control on atherosclerotic plaque stability remains unclear.

It has been established that atherosclerotic plaque rupture is a critical event triggering thrombus formation and subsequent acute coronary syndrome (Libby 1995, Libby \& Theroux 2005). Pathologically, plaques that are prone to rupture consist of a larger intimal lesion and an increased number of macrophages. Analysis of gene expression in atherosclerotic plaques showed that when compared with stable plaques, vulnerable plaques have higher expression of matrix metalloproteinases (MMPs) that weaken atherosclerotic plaques (Richardson et al. 1989, Falk et al. 1995). Autopsy studies have demonstrated that atherosclerosis in diabetic patients is more extensive and accelerated than that in nondiabetic patients (Jarrett 1981). Furthermore, studies have also shown that atherosclerotic lesions in diabetic patients were more vulnerable as they had larger intimal lesions, more 
macrophage infiltration and thrombosis compared with those in nondiabetic patients (Moreno et al. 2000). Given that plaque rupture is a key event in triggering cardiovascular events, these studies suggest that glycemic control may decrease the mortality and morbidity of cardiovascular disease in diabetic patients by stabilizing atherosclerotic plaques.

MMPs are a family of zinc-dependent endopeptidases capable of degrading extracellular matrix components and play an essential role in vascular tissue remodeling (Galis \& Khatri 2002). It is known that MMPs are involved in plaque destabilization and ultimate plaque rupture (Galis \& Khatri 2002). Among various MMPs, plasma MMP9 has been associated with the presence of coronary atherosclerosis and is an independent risk factor for atherothrombotic events (Loftus et al. 2001, Blankenberg et al. 2003, Montaner et al. 2003). Furthermore, it has been reported that the plasma MMP9 level in patients with both diabetes and coronary artery disease was significantly higher than that in age-, sex-, and body mass index-matched patients with coronary artery disease alone (Marx et al. 2003), indicating an important role of MMP9 in diabetes-associated atherosclerosis. Given the importance of MMP9 in diabetes-associated atherosclerosis, we proposed that glycemic control might reduce cardiovascular events by inhibiting MMP9 expression in atherosclerotic plaques and thus stabilizing atherosclerotic plaques.

In this study, we employed apolipoprotein E-deficient (apoE-/-) mice with streptozotocin (STZ)-induced diabetes and studied the effect of insulin treatment on diabetes-increased intimal lesion and expression of MMP9 and interleukin (IL) 6, a stimulator for MMP9, in atherosclerotic lesions.

\section{Materials and Methods}

\section{Animals and diets}

Male C57BL/6 mice and apoE - / - mice were purchased from Jackson Laboratory (Bar Harbor, ME, USA) and housed at the animal facility in the Veterans Affairs Medical Center in Charleston, SC, USA. The animal protocol was approved by the Institutional Animal Care and Use Committee (IACUC). The C57BL/6 mice served as nonatherosclerosis control (group 1) and apoE - / - mice were randomly divided into three groups (groups 2-4). All mice were maintained on a $12 \mathrm{~h}$ light:12 h darkness cycle in a pathogen-free environment and had ad libitum access to regular chow (Harlan Teklad 8604, Indianapolis, IN, USA) and water. At 12 weeks of age, the mice in groups 3 and 4 were rendered diabetic by i.p. administration of single dose of STZ (Sigma-Aldrich), $200 \mathrm{mg} / \mathrm{kg}$ in citrate buffer, $\mathrm{pH} \mathrm{4.5}$. At 4 weeks after the STZ treatment, the mice in group 4 were treated with longacting Lantus insulin (Aventis Pharmaceuticals, Bridgewater, NJ, USA) by daily s.c. injection of 1 unit/mouse for 20 weeks.

\section{Metabolic measurements}

Blood samples were obtained under the fasting condition, and glucose level was determined using a Precision QID glucometer (MediSense, Inc., Bedford, MA, USA). Plasma cholesterol and triglycerides were measured by enzymatic methods (Wako Diagnostics, Richmond, VA, USA).

\section{Histological analysis of atherosclerotic lesions}

The tissues of aortic root were dissected and frozen in Trisbuffered saline (TBS) freezing media and sectioned with a cryostat. Sections with $5 \mu \mathrm{m}$ thickness were mounted on slides before being placed in 95\% ethanol for $10 \mathrm{~min}$ and washed in PBS. Fixed hydrated slides were stained with Harris modified hematoxylin (Fisher Scientific, Pittsburgh, $\mathrm{PA}$, USA) for $3 \mathrm{~min}$ and then rinsed in deionized water. Staining of hematoxylin was developed in tap water for $5 \mathrm{~min}$, and the slides were dipped in acid ethanol to stop the development. Slides were stained with eosin in Harleco 1\% alcohol solution (EMD Chemicals, Gibbstown, NJ, USA) for $30 \mathrm{~s}$. Slides were placed in Coplin jars with 95\% ethanol three times for $5 \mathrm{~min}$ each, and then this was repeated with $100 \%$ ethanol. Slides were further dehydrated in $99 \cdot 5 \%$ xylene (Sigma-Aldrich) and mounted in xylene-based Cytoseal-XYL mounting media (Fisher Scientific). Photomicrographs of tissue sections were taken using a Nikon Eclipse 90i digital microscope with the NIS Elements 3.10. image analysis system (Nikon Instruments, Inc., Lewisville, TX, USA). The area of intima was presented as the percentage of the total aortic area including intima, media, and lumen (Collins et al. 2001).

\section{Immunohistochemistry for MMP9 and IL6}

Frozen tissue sections were exposed to $2 \%$ normal goat serum for $20 \mathrm{~min}$. Primary monoclonal antibodies to MMP9 or IL6 (Santa Cruz Biotechnology, Inc., Santa Cruz, CA, USA) were applied to the sections at 1:40-1:100 dilutions for $45 \mathrm{~min}$. Sections were then incubated with secondary biotinylated antibody from the ABC Elite kit (Vector Laboratories, Burlingame, CA, USA) for $30 \mathrm{~min}$ at 1:250 dilutions. After being exposed to avidin-biotin solution from the ABC Elite kit for $30 \mathrm{~min}$, slides with the tissue sections were placed in 30\% methanol peroxide. The tissue sections were covered with diaminobenzidine peroxidase substrate solution from the Impact DAB kit (Vector Laboratories) for 3 min. Slides were rinsed in water and counterstaining was performed with hematoxylin. Slides were then dehydrated using increasing concentrations of ethanol and xylene and mounted. Staining with normal rabbit IgG was used as a negative control. Photomicrographs of atherosclerotic lesions with positively immunostained cells were taken using the computer-based image analysis system as described above in the histological study. 


\section{Image analysis}

Images were analyzed using the Photoshop software (version 10; Adobe Systems). The method of using the 'Similar' feature to select a particular color staining on a digitized immunohistochemical image has previously been described in detail (Lehr et al. 1997). Briefly, a standard was created by selecting an area of $0.5 \times 0.5 \mathrm{~cm}$ from a tissue section that had desired brown color from immunostaining. The cursor of the Magic Wand tool was clicked on the standard to make a selection and the area of the standard was highlighted. To specify how broad a range of color the Magic Wand tool should include in the selection, the tolerance value in the Magic Wand Options palette was set to 100. Using the 'Similar' command, all the areas with the brown color that is similar to the standard on an image being determined were highlighted. The quantification was done using the histogram command in the Image menu, which showed the pixels of the highlighted area. The extent of the positive immunostaining was presented as average pixels per cross-section of atherosclerotic lesions.

\section{Isolation of RNA from aortic tissue}

The vascular tissues of aortic root were homogenized in TRIzol solution (Invitrogen) on ice using an ultrasonic processor (GE130, Sonics \& Materials, Inc., Newtown, CT, USA). After homogenization, $0.75 \mathrm{ml}$ homogenate was mixed with $0.2 \mathrm{ml}$ chloroform and centrifuged at $12000 \mathrm{~g}$ for $15 \mathrm{~min}$. The aqueous phase was removed, mixed with equal volume of $70 \%$ ethanol, and loaded into the RNeasy column (Qiagen) for RNA isolation using the RNeasy mini kit by following the instructions from the manufacturer.

\section{Quantitative real-time PCR}

First-strand complementary DNA (cDNA) was synthesized using the iScript cDNA synthesis kit (Bio-Rad Laboratories) using $20 \mu \mathrm{l}$ reaction mixture containing $0 \cdot 25 \mu \mathrm{g}$ total RNA, $4 \mu \mathrm{l} 5 \times$ iScript reaction mixture, and $1 \mu \mathrm{l}$ iScript reverse transcriptase. The complete reaction was cycled for $5 \mathrm{~min}$ at $25^{\circ} \mathrm{C}, 30 \mathrm{~min}$ at $42{ }^{\circ} \mathrm{C}$, and $5 \mathrm{~min}$ at $85^{\circ} \mathrm{C}$ using a PTC-200 DNA Engine (MJ Research, Waltham, MA, USA). The reverse transcription (RT) reaction mixture was then diluted
1:10 with nuclease-free water and used for PCR amplification of cDNA in the presence of the primers. The Beacon designer software (PREMIER Biosoft International, Palo Alto, CA, USA) was used for primer designing (Table 1). Primers were synthesized by Integrated DNA Technologies, Inc. (Coralville, IA, USA), and real-time PCR was performed in duplicate using $25 \mu \mathrm{l}$ reaction mixture containing $1 \cdot 0 \mu \mathrm{l}$ RT mixture, $0 \cdot 2 \mu \mathrm{M}$ both primers, and $12 \cdot 5 \mu \mathrm{l}$ Q SYBR Green Supermix (Bio-Rad Laboratories). Real-time PCR was run in the iCycler real-time detection system (Bio-Rad Laboratories) with a two-step method. The hot-start enzyme was activated $\left(95{ }^{\circ} \mathrm{C}\right.$ for $3 \mathrm{~min}$ ), and cDNA was then amplified for 40 cycles consisting of denaturation at $95{ }^{\circ} \mathrm{C}$ for $10 \mathrm{~s}$ and annealing/ extension at $53{ }^{\circ} \mathrm{C}$ for $45 \mathrm{~s}$. A melt curve assay was then performed $\left(55^{\circ} \mathrm{C}\right.$ for $1 \mathrm{~min}$ and then temperature was increased by $0.5{ }^{\circ} \mathrm{C}$ every $10 \mathrm{~s}$ ) to detect the formation of primer-derived trimers and dimers. Glyceraldehyde-3-phosphate dehydrogenase (GAPDH) was used as a control. Data were analyzed using the iCycler $\mathrm{iQ}$ software. The average starting quantity (SQ) of fluorescence units was used for analysis. Quantification was calculated using the SQ of targeted cDNA relative to that of GAPDH cDNA in the same sample.

\section{Cell culture}

U937 mononuclear cells (Sundstrom \& Nilsson 1976) were purchased from American Type Culture Collection (Manassas, VA, USA). The cells were cultured in a $5 \% \mathrm{CO}_{2}$ atmosphere in RPMI 1640 medium (GIBCO, Invitrogen Corp.) containing different glucose concentrations (5-25 mM), 10\% FCS, 1\% MEM nonessential amino acid solution, and $0.6 \mathrm{~g} / 100 \mathrm{ml} \mathrm{HEPES}$.

\section{ELISA}

MMP9 and tissue inhibitor of metalloproteinase (TIMP)1 in conditioned medium were quantified using sandwich ELISA kits according to the protocol provided by the manufacturer (R\&D System, Minneapolis, MN, USA).

\section{Statistical analysis}

Data are presented as mean \pm s.D. Comparison between treatments was performed by the one-way ANOVA. A value of $P<0 \cdot 05$ was considered significant.

Table 1 Primer sequences for real-time PCR

\section{Genes}

MMP9

IL6

$T N F \alpha$

$M C P 1$

$M-C S F$

$G A P D H$
5 ' Primer sequence

GCTGACTACGATAAGGACGG
ATGAAGTTCCTCTCTGCAAGAGACT
TTCTGTCTACTGAACTTCGGGGTGATCGGTCC
CTTCTGGGCCTGCTGTTCA
CATCCACGCTGCGTGAAG
GDDTTCCGTGTTCCTACC

$3^{\prime}$ Primer sequence

CTCAAAGATGAACGGGAACAC

CACTAGGTTTGCCGAGTAGATCTC

GTATGAGATAGCAAATCGGCTGACGGTGTGGG

CCAGCCTACTCATTGGGATCA

GGGATTCGGTGTCGCAATAT

GCCTGCTTCACCACCTTC 


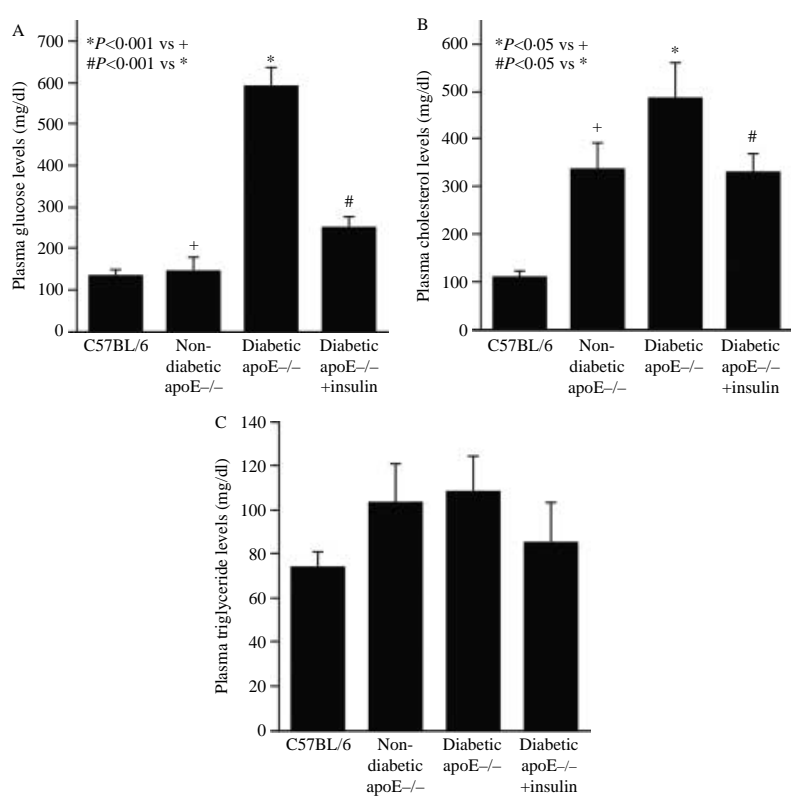

Figure 1 Plasma glucose (A), cholesterol (B), and triglyceride levels (C) in nondiabetic and diabetic mice. The plasma glucose, cholesterol, and triglycerides in C57BL/6 $(n=8)$, nondiabetic apoE $-/-(n=8)$, diabetic apoE $-/-(n=10)$, and insulin-treated diabetic apoE $-/-$ mice $(n=10)$ were quantified at the end of the study.

\section{Results}

\section{The effect of insulin treatment on the metabolic parameters}

The plasma levels of glucose, cholesterol and triglycerides were determined in nondiabetic and diabetic mice treated with or without insulin. Results showed that glucose level in nondiabetic apoE $-/-$ mice was similar to that in C57BL/6 mice. As expected, glucose level was markedly increased in STZ-treated mice, but the increase was reduced by insulin treatment by $76 \%$ (Fig. 1A). Lipid analysis showed that the total cholesterol level was increased in nondiabetic apoE $-/-$ mice by threefold compared with that in $\mathrm{C} 57 \mathrm{BL} / 6$ mice. Induction of diabetes in apoE $-/-$ mice further increased total cholesterol level by $43 \%$, but insulin treatment abolished the effect of diabetes on cholesterol (Fig. 1B). For triglycerides, no difference was found among four groups (Fig. 1C). Taken together, these data indicate that insulin treatment effectively reduced diabetes-increased hyperglycemia and cholesterol.

\section{Reduction in intimal lesions by insulin treatment}

The image analysis of aortic atherosclerotic lesions showed that C57BL/6 mice had little neointima formation in the aortic origin (Fig. 2A, panel a and B), whereas nondiabetic apoE - / - mice had well-developed atherosclerotic plaques (Fig. 2A, panel $\mathrm{b}$ and $\mathrm{B}$ ). In diabetic apoE $-/-$ mice, intimal lesions were more extensive than those in nondiabetic
apoE $-/-$ mice $(33 \pm 7$ vs $10 \pm 6 \%, P<0 \cdot 01$; Fig. $2 \mathrm{~A}$, panel $\mathrm{c}$ and B). Furthermore, insulin treatment in diabetic mice significantly attenuated diabetes-increased intimal lesions by $60 \%$ (Fig. 2A, panel d and B).

Inhibition of MMP9, but not TIMP1, by insulin treatment

The expression of MMP9 in the intimal lesions of atherosclerotic plaques was detected by immunohistochemistry. Results showed that MMP9 was markedly increased in the intimal lesions in diabetic apoE $-/-$ mice compared with that

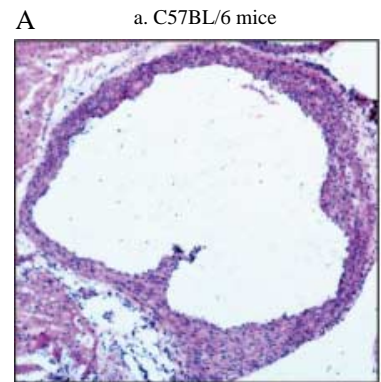

c. Diabetic apoE-/-mice

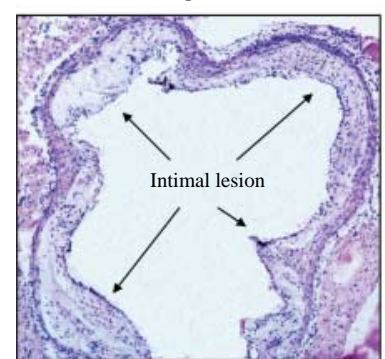

B

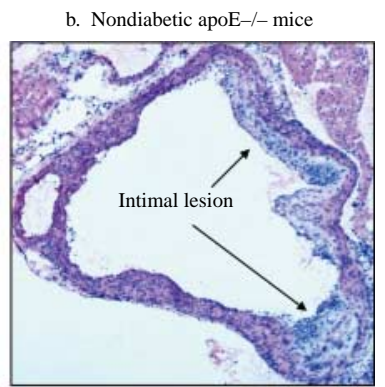

d. Diabetic apoE-/- mice with insulin treatment

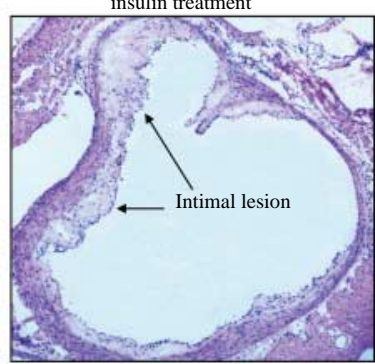

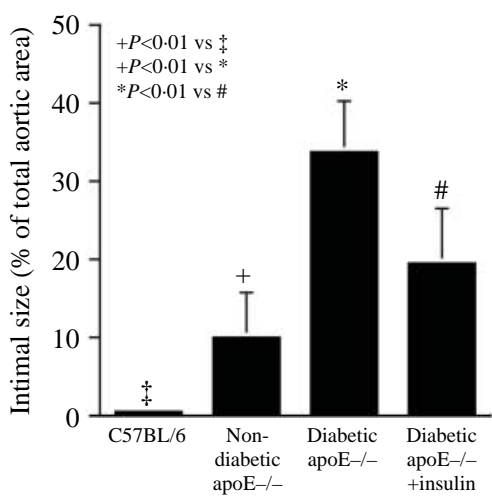

Figure 2 Inhibition of diabetes-increased atherosclerotic intimal lesion by insulin treatment. The vascular tissues of the aortic roots were dissected from $\mathrm{C} 57 \mathrm{BL} / 6$ mice, apoE $-/-$ mice with or without STZ-induced diabetes, and diabetic apoE-/- mice treated with insulin. Frozen sections were made from the tissues and stained with hematoxylin and eosin. (A) Representative photomicrographs of atherosclerotic plaques of $\mathrm{C} 57 \mathrm{BL} / 6$ (a), nondiabetic apoE $-/-$ mice (b), diabetic apoE $-/-$ mice (c), and diabetic apoE $-/-$ mice treated with insulin (d). (B) Quantification of intimal lesion area of atherosclerotic plaques. The intimal lesions were quantified and presented as the percentage of the total aortic area including intima, media, and lumen $(n=8-10)$. 


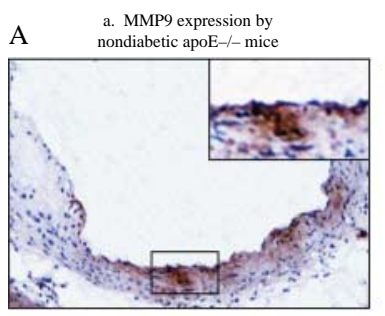

c. MMP9 expression by diabetic apoE-/- mice
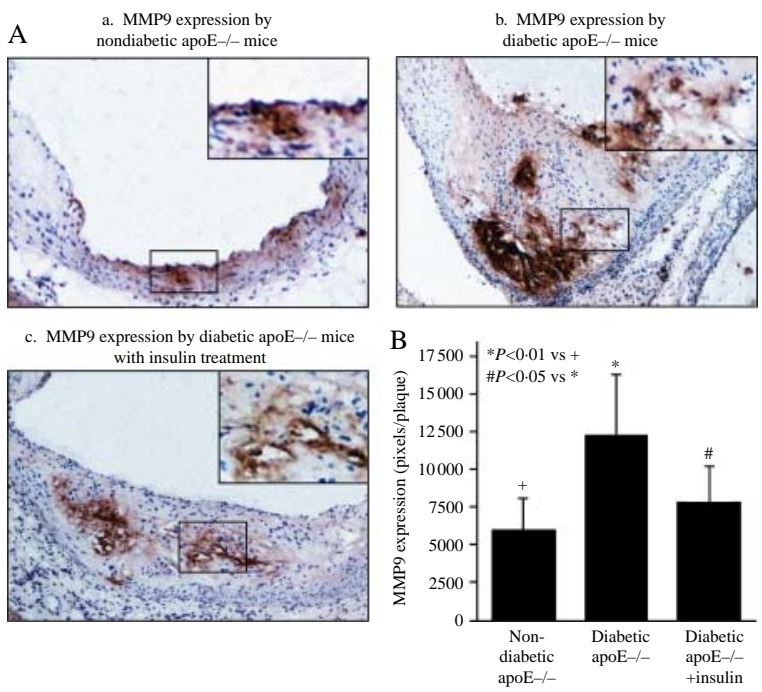

Figure 3 Inhibition of diabetes-increased MMP9 quantification in atherosclerotic plaques by insulin treatment. MMP9 expression in atherosclerotic plaques was detected by immunostaining and presented as pixels per plaque. (A) Representative photomicrographs of MMP9 immunostaining of atherosclerotic plaques from nondiabetic apoE $-/-$ mice (a), diabetic apoE $-/-$ mice (b), and diabetic apoE - / - mice treated with insulin (c). The detailed images in the areas as indicated are enlarged in the insets.

(B) Quantification of MMP9 in atherosclerotic plaques $(n=8-10)$.

in nondiabetic apoE $-/-$ mice (Fig. 3A, panels a and $b$ and B). However, MMP9 expression was significantly reduced in diabetic apoE $-/-$ mice treated with insulin (Fig. 3A, panel $\mathrm{c}$ and B). MMP9 expression was localized not only in the lipid core (Fig. 3A, insets of panel b and c), but also in the area near surface of atherosclerotic plaque (Fig. 3A, inset of panel a). Although the expression of MMP13 and MMP2 in atherosclerotic plaques was also increased in diabetic mice, insulin treatment did not inhibit the expression (data not shown), suggesting that insulin treatment had a specific inhibition on MMP9 expression. Furthermore, although TIMP1 expression appeared to be increased in diabetic mice with insulin treatment when compared with that in nondiabetic and diabetic mice without insulin treatment, the increase did not reach significance statistically (Fig. 4).

In support of the findings from the above immunohistochemical studies, experiments using quantitative real-time PCR showed that MMP9 mRNA expression in vascular tissues of the aortic root was significantly increased in diabetic apoE - / - mice compared with that in nondiabetic mice, and that the increase was completely abolished by insulin treatment (Fig. 5).

\section{Insulin treatment inhibits IL6 expression in} atherosclerotic plaques

It is known that IL6 is expressed in atherosclerotic plaques and involved in MMP expression (Li et al. 2010). To determine the effect of diabetes and insulin treatment on the expression of
IL6, we assessed IL6 expression in atherosclerotic lesions by immunohistochemistry. Results showed that diabetes significantly increased IL6 expression (Fig. 6A, panels a and b and $\mathrm{B})$, but insulin treatment markedly attenuated diabetesincreased IL6 expression (Fig. 6A, panel $\mathrm{c}$ and B). Similar findings were observed from studies using quantitative real-time PCR technique, which showed a significant increase in IL6 mRNA in the vascular tissues of aortic root of diabetic mice compared with that of nondiabetic mice and the attenuation of IL6 upregulation by insulin treatment (Fig. 7A).

In addition to IL6, we also determined mRNA expression of tumor necrosis factor (TNF) $\alpha$, monocyte chemotactic protein (MCP) 1, macrophage colony-stimulating factor (M-CSF), and CD11B, which are all involved in macrophage recruitment and plaque destabilization (Gray \& Shankar 1995, Yakubenko et al. 2008, McKellar et al. 2009, Yadav et al. 2010, Brocheriou et al. 2011). Results (Fig. 7B) showed that diabetes increased TNF $\alpha$ and MCP1 mRNA expression, but insulin treatment only inhibited MCP1 mRNA expression. Diabetes decreased $M$-CSF mRNA expression and insulin treatment did not change the effect of diabetes. In addition, we found that diabetes had no effect on CD11B mRNA expression (data not shown).

\section{Reduction in glucose attenuates IL6-stimulated MMP9 by mononuclear cells in vitro}

Figure 3 showed that insulin treatment reduced MMP9 expression in atherosclerotic plaques in diabetic mice. Given
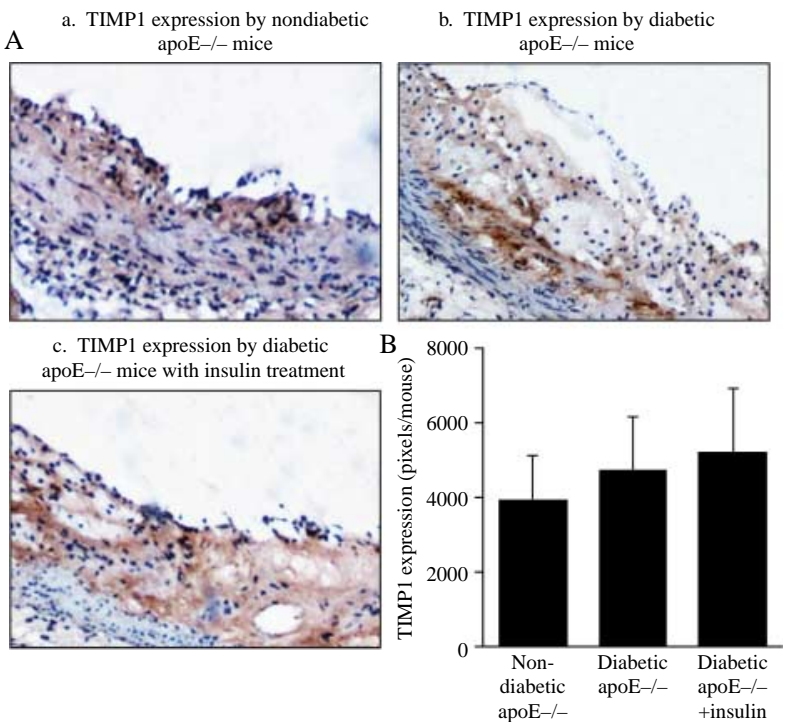

Figure 4 Effect of diabetes and insulin treatment on TIMP1 quantification in atherosclerotic plaques. TIMP1 protein expression in atherosclerotic plaques was detected by immunostaining and presented as pixels per plaque. (A) Representative photomicrographs of TIMP1 immunostaining of atherosclerotic plaques from nondiabetic apoE - / - mice (a), diabetic apoE - /- mice (b), and diabetic apoE $-/-$ mice treated with insulin (c). (B) Quantification of TIMP1 protein in atherosclerotic plaques $(n=8-10)$. 


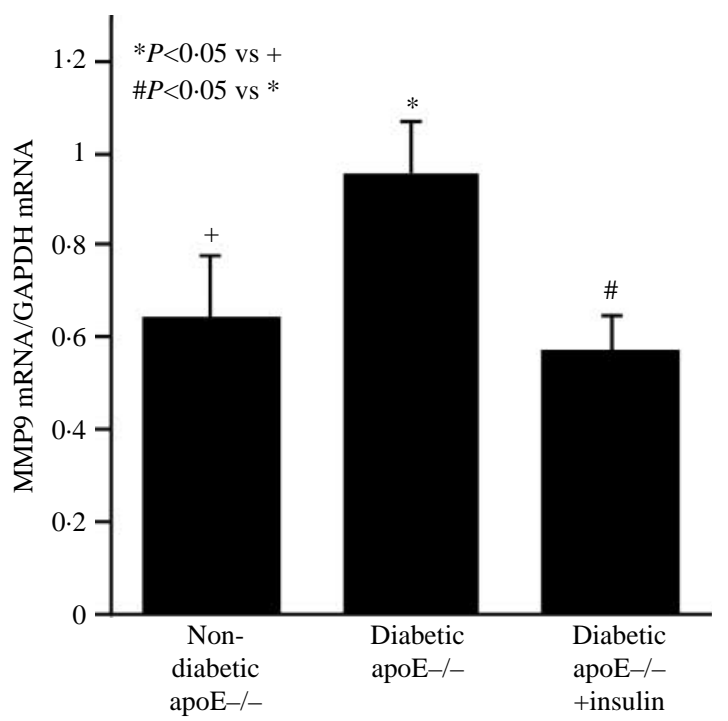

Figure 5 Inhibition of diabetes-increased MMP9 mRNA quantification in atherosclerotic plaques by insulin treatment. Total RNA was isolated from vascular tissue of aortic root of nondiabetic apoE $-/-$ mice, diabetic apoE $-/-$ mice, and diabetic apoE $-/-$ mice treated with insulin and subjected to real-time PCR to quantify $M M P 9$ and GAPDH mRNA. Data presented are mean \pm s.D. of the ratio of MMP9 mRNA versus GAPDH mRNA $(n=7)$.

that insulin treatment lowered serum glucose in diabetic mice, we hypothesized that glucose reduction downregulates MMP9 expression in atherosclerotic plaques. To test this hypothesis, we pre-exposed U937 cells with different concentrations of glucose and then treated with IL6, a stimulator for MMP9 expression. After the treatment, MMP9 in the culture medium was quantified by ELISA. Results showed that IL6-increased MMP9 secretion by cells pre-exposed to 25,15 , and $5 \mathrm{mM}$ of glucose was $1.8,1 \cdot 2$, and $0.8 \mathrm{ng} / \mathrm{ml}$ respectively (Fig. 8A), suggesting that reduction in glucose from 25 or 15 to $5 \mathrm{mM}$ significantly attenuated IL6-increased MMP9 secretion. These in vitro findings suggest that glucose reduction by insulin treatment may play an important role in insulin treatmentattenuated MMP9 expression in atherosclerotic lesions.

The effect of high glucose $(25 \mathrm{mM})$ on TIMP1 was also studied. Results showed that high glucose alone had no effect on TIMP1 secretion compared with normal glucose $(5 \mathrm{mM}$; Fig. 8B). Furthermore, the combination of high glucose and IL6 also had no significant stimulation on TIMP1 secretion compared with the combination of normal glucose and IL6 (Fig. 8B), indicating that reduction in glucose from 25 to $5 \mathrm{mM}$ does not significantly decrease TIMP1 secretion by mononuclear cells in response to IL6.

\section{Effect of insulin treatment on macrophage content in atherosclerotic lesions}

Macrophages in atherosclerotic plaques were detected by immunostaining with antibody against mouse CD68, a surface marker for mouse macrophages. Results showed that diabetes increased macrophage content markedly (Fig. 9A, panels $\mathrm{a}$ and $\mathrm{b}$ and $\mathrm{B}$ ). Interestingly, insulin treatment did not reduce macrophage content (Fig. 9A, panel $\mathrm{c}$ and $\mathrm{B}$ ).

\section{Discussion}

Clinical trials have shown that a good glycemic control in patients with type 1 diabetes significantly reduces cardiovascular disease mortality and morbidity (Soedamah-Muthu \& Stehouwer 2005). To understand how glycemic control in diabetic subjects reduces cardiovascular events, we focused on the effect of glycemic control on atherosclerotic lesions and gene expression that affects plaque stability in this study. With long-acting Lantus insulin, we successfully reduced hyperglycemia in diabetic apoE $-/-$ mice. The metabolic studies showed that STZ injection markedly increased plasma glucose level by threefold (591 vs $145 \mathrm{mg} / \mathrm{dl}$ ), but insulin treatment led to a $76 \%$ reduction in diabetes-increased glucose. Therefore, this study established an effective treatment on hyperglycemia using long-acting insulin in a mouse model for atherosclerosis and diabetes.

Previous studies have shown that induction of diabetes in apoE - / - mice increases not only plasma glucose but also cholesterol (Park et al. 1998, Tse et al. 1999, Candido et al. 2004, Hayek et al. 2005). However, the levels of cholesterol increase varied remarkably between these studies. For
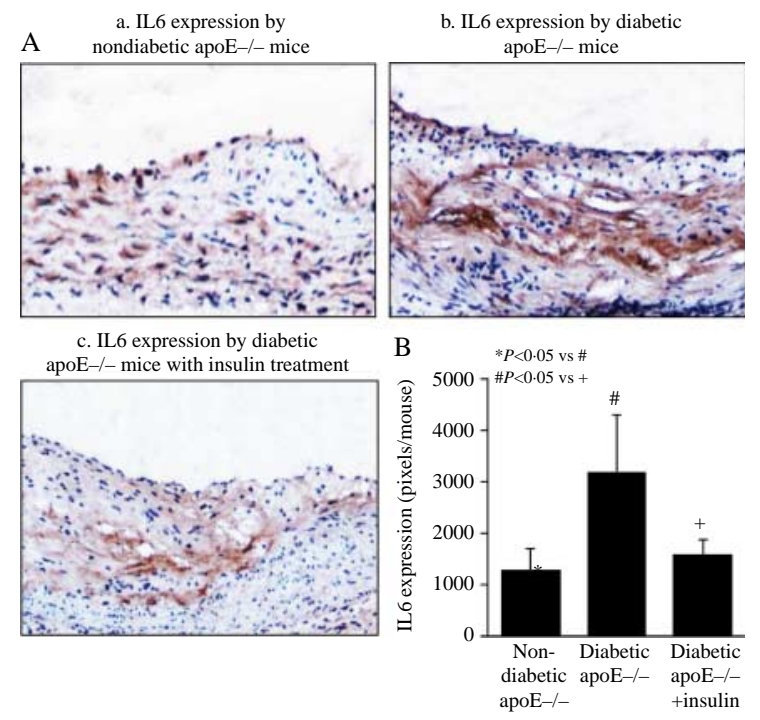

Figure 6 Inhibition of diabetes-increased IL6 expression in atherosclerotic plaques by insulin treatment. IL6 protein expression in atherosclerotic plaques was detected by immunostaining and presented as pixels per plaque. (A) Representative photomicrographs of IL6 immunostaining of atherosclerotic plaques from nondiabetic apoE-/- mice (a), diabetic apoE-/mice (b), and diabetic apoE $-/-$ mice treated with insulin (c).

(B) Quantification of IL6 protein expression in atherosclerotic plaques $(n=8-10)$. 


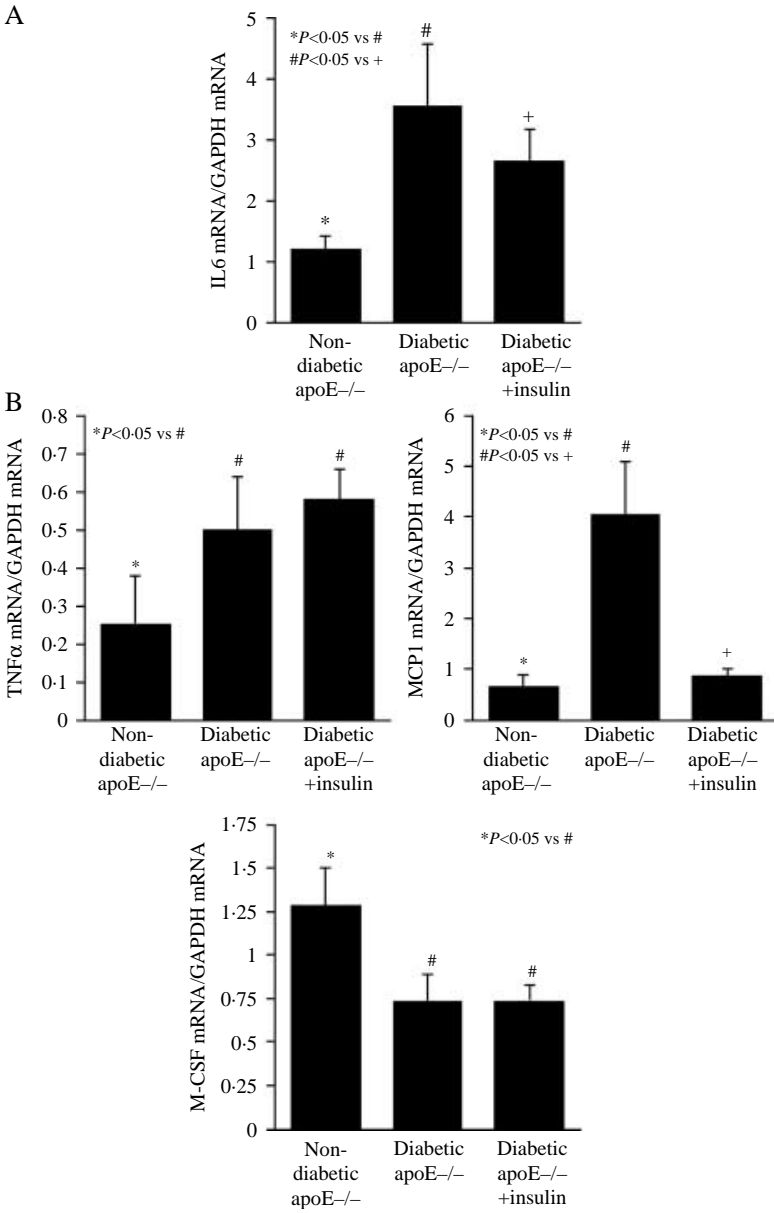

Figure 7 Effect of insulin treatment on IL6 (A), TNF $\alpha, M C P 1$, and $M-C S F$ mRNA (B) expression in atherosclerotic plaques. Total RNA was isolated from vascular tissue of aortic root of nondiabetic apoE $-/-$ mice, diabetic apoE $-/-$ mice, and diabetic apoE-/mice treated with insulin and subjected to real-time PCR to quantify IL6, TNF $, M C P 1, M-C S F$, and GAPDH mRNA. Data presented are mean \pm s.D. of the ratio of MMP9 mRNA versus GAPDH mRNA $(n=7)$.

example, the study by Park et al. (1998) reported that the average cholesterol level in apoE $-/-$ mice with STZinduced diabetes was $1359 \mathrm{mg} / \mathrm{dl}$, which was $2 \cdot 7$-fold of that in nondiabetic apoE $-/-$ mice, while the study by Hayek et al. (2005) reported that the average cholesterol level in diabetic apoE $-/-$ mice was $545 \mathrm{mg} / \mathrm{dl}$, only $13 \%$ increase (not significant) over that in nondiabetic apoE $-/-$ mice. Interestingly, the glucose levels in these two studies were similar, $350-500 \mathrm{mg} / \mathrm{dl}$ in the study of Park et al. and 415 $\pm 223 \mathrm{mg} / \mathrm{dl}$ in the study of Hayek et al., suggesting that the large difference in cholesterol increase is not due to glucose increases. An apparent difference between these two studies was the method in STZ treatment. Park et al. treated mice with six daily i.p. injections of STZ at the dose of $55-65 \mathrm{mg} / \mathrm{kg}$, whereas Hayek et al. treated with only one injection at the dose of $200 \mathrm{mg} / \mathrm{kg}$. It remains unclear whether repeated injection with lower doses of STZ increased plasma cholesterol to a greater extent compared with a single injection with higher dose. In this study, we employed the method of Hayek et al. in STZ treatment, and found that STZ treatment led to a threefold increase in glucose with a moderate $43 \%$ increase in cholesterol. We also found that insulin treatment led to an effective glycemic control and significant reduction in cholesterol.

In humans, it is known that hyperglycemia is not associated with hypercholesterolemia, and glycemic control is not associated with a decrease in cholesterol level. However, an inverse relationship between $\mathrm{HbA} 1 \mathrm{C}$ and high-density lipoprotein (HDL) cholesterol levels in type 2 diabetic patients has been reported (Khan et al. 2007). Gatti et al. (2009) have recently reported that after adjustments for obesity and hypertriglyceridemia in multivariate analysis, poor glycemic control was found to be an independent risk factor for low HDL cholesterol in patients with type 2 diabetes. These studies, therefore, indicate that hyperglycemia in humans also affects cholesterol metabolism. The difference is that hyperglycemia in apoE $-/-$ mouse increases total cholesterol level, whereas hyperglycemia in humans decreases HDL cholesterol. Given the critical role of cholesterol in atherosclerosis, it is likely that both hyperglycemia and hypercholesterolemia in apoE $-/-$ mice in this study contributed to diabetes-promoted progression of atherosclerosis. On the other hand, the reduction in both glucose and cholesterol by insulin treatment is also likely responsible for the attenuation of diabetes-promoted atherosclerosis.

It has been shown that apoE $-/-$ mice on a normal chow diet first manifest foam-cell lesions in aorta at 8 weeks of age, and develop advanced and complex lesions after 15 weeks (Nakashima et al. 1994). In this study, apoE - / mice were grown for 32 weeks, and therefore we considered that these mice had developed advanced atherosclerotic plaques. It is known that large intimal lesion is one of the features for unstable plaques in the advanced stage
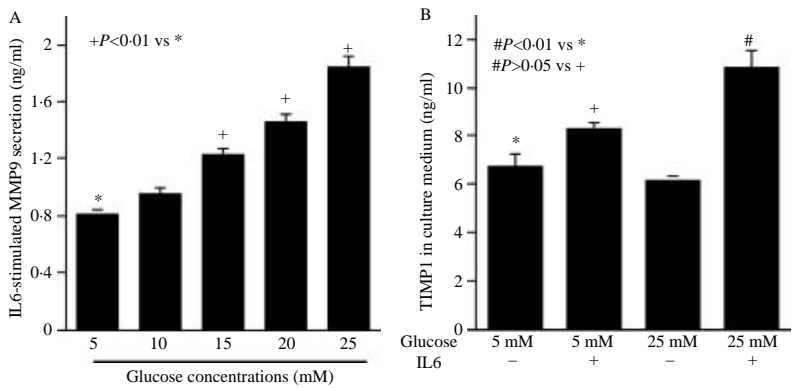

Figure 8 Effect of glucose concentration on MMP9 and TIMP1 secretion by U937 mononuclear cells in response to IL6. (A) U937 mononuclear cells were pre-exposed to different concentrations of glucose (5-25 mM) for 2 days and then treated with $100 \mathrm{ng} / \mathrm{ml} \mathrm{IL6}$ for $24 \mathrm{~h}$. MMP9 released by cells into medium was quantified by ELISA. (B) U937 cells were pre-exposed to normal ( $5 \mathrm{mM}$ ) or high glucose $(25 \mathrm{mM})$ for 2 days and then treated with or without $100 \mathrm{ng} / \mathrm{ml}$ IL6 for $24 \mathrm{~h}$. After the treatment, TIMP1 in culture medium was quantified by ELISA. 


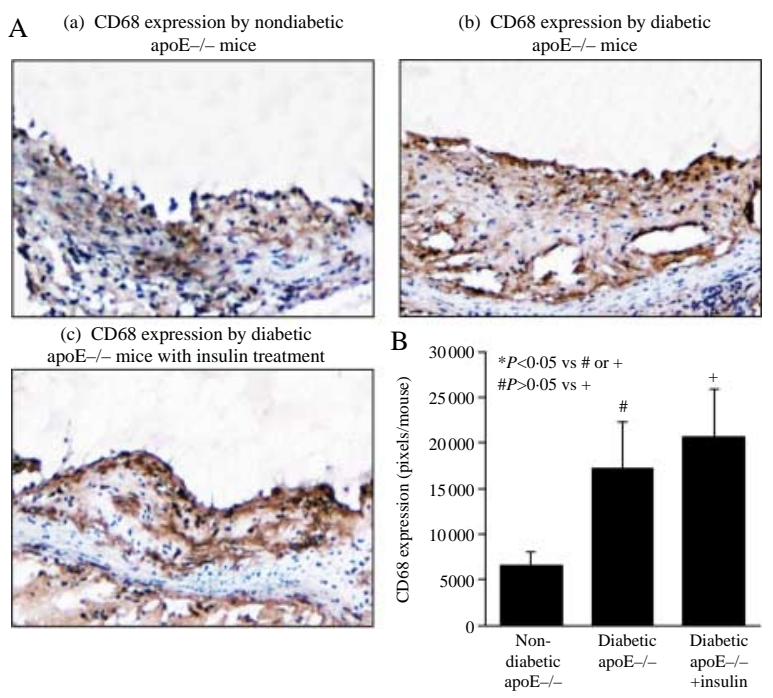

Figure 9 Effect of diabetes and insulin treatment on CD68 expression in atherosclerotic plaques. CD68 protein expression in atherosclerotic plaques was detected by immunostaining and presented as pixels per plaque. (A) Representative photomicrographs of CD68 immunostaining of atherosclerotic plaques from nondiabetic apoE - / - mice (a), diabetic apoE - / - mice (b), and diabetic apoE $-/-$ mice treated with insulin (c). (B) Quantification of CD68 protein expression in atherosclerotic plaques $(n=8-10)$.

(Richardson et al. 1989, Falk et al. 1995). Autopsy studies have shown that diabetic patients have more extensive atherosclerotic plaques in coronary arteries than nondiabetic patients (Jarrett 1981, Moreno et al. 2000). Consistent with these clinical findings, we observed that diabetes increased intimal lesion area in apoE $-/-$ mice by $3 \cdot 3$-fold when compared with nondiabetic mice. This is a striking finding and has been reported consistently by other laboratories. For example, Candido et al. (2004) showed that the atherosclerotic plaque area in apoE $-/-$ mice that had diabetes for 20 weeks was fivefold of that in nondiabetic mice. Park et al. (1998) also reported similar. In this study, in addition to the effect of hyperglycemia on intimal lesions, we demonstrated that insulin treatment significantly reduced diabetes-increased lesion size. Thus, this study further underscores an important role of hyperglycemia in atherosclerosis.

It has been well established that MMPs are upregulated in unstable plaques and play a critical role in plaque destabilization (Galis \& Khatri 2002). It has been also reported that although TIMPs that inhibit MMP activities are upregulated in unstable plaques as well, MMPs are stimulated to a much higher extent than TIMPs, leading to an imbalance between MMP and TIMP expression (Galis et al. 1994, Galis \& Khatri 2002). In this study, we demonstrated that diabetes significantly increased the expression of MMP9, but not of TIMP1, in atherosclerotic plaques. Furthermore, we demonstrated that insulin treatment significantly reduced diabetesincreased MMP9 expression but had no effect on TIMP1 expression. In support of these in vivo observations, our in vitro studies also showed that high glucose augmented IL6-stimulated MMP9 secretion in a concentrationdependent manner. Reduction in glucose concentration from 25 to $5 \mathrm{mM}$ decreased IL6-stimulated MMP9 secretion by $56 \%$. Taken together, these studies showed that induction of diabetes in apoE $-/-$ mice increased the ratio of MMP9 versus TIMP1 expression, but insulin treatment led to a rebalance between MMP9 and TIMP1 expression.

The mechanisms involved in the stimulation of MMP9 by high glucose have been explored in recent years (Bai et al. 2005, Lee et al. 2007, Nareika et al. 2009). Our laboratory showed that high glucose increased the expression of c-Jun and c-Fos, which are the major subunits of AP-1 transcription factor (Li et al. 2010). Given that AP1 is a key transcription factor for MMP9 expression (Aljada et al. 2001), it is most likely that high glucose stimulates MMP9 expression by increasing AP1 level. Furthermore, under the pathological conditions such as diabetes, multiple stimulators such as high glucose and proinflammatory cytokines are present in atherosclerotic plaques. All these stimulators are likely to act in concert to stimulate MMP9 expression. Our findings on the coordinated stimulation on MMP9 by high glucose and IL6 (Fig. 8A) have provided evidence indicating the effect of multiple stimulators on MMP9 upregulation. Since IL6 also increased c-Jun and c-Fos expression ( $\mathrm{Li}$ et al. 2010), it is likely that high glucose and IL6 synergistically increase MMP9 expression by their coordinate action on AP1 activity.

In addition to the stimulation of MMP9 expression by IL6 and other proinflammatory cytokines, previous studies have shown that nitric oxide (NO) inhibited MMP2 and MMP9 expression by smooth muscle cells (Gurjar et al. 1999) and enhancement of eNOS expression in rat heart reduced MMP9 expression (Liu et al. 2011). Thus, MMP9 expression is associated with endothelial dysfunction. Given that chronic exposure to high glucose reduces NO generation in endothelial cells, probably by impairing phospholipaseC-mediated $\mathrm{Ca}^{2+}$ signaling due to excess protein kinase $\mathrm{C}$ activation (Tang \& $\mathrm{Li} \mathrm{2004),} \mathrm{it} \mathrm{is} \mathrm{obvious} \mathrm{that} \mathrm{diabetes}$ downregulates MMP9 expression via endothelial dysfunction and subsequent reduction in $\mathrm{NO}$ production from endothelial cells.

Increased macrophage content is another important feature for unstable plaques (Moreno et al. 2000). Pathological studies have shown that diabetes is associated with an increase in macrophage number in atherosclerotic plaques (Jarrett 1981), suggesting that the increased macrophage content in plaque destabilization in diabetes may contribute to plaque instability. Consistent with these reports, this study showed that macrophage content in atherosclerotic plaques as detected by CD68 immunostaining was significantly increased in diabetic apoE $-/-$ mice. Surprisingly, our data showed that insulin treatment did not reduce diabetes-increased macrophage content. It is possible that in addition to hyperglycemia, other diabetes-associated factors may also influence macrophage content in atherosclerotic plaques, and glycemic control alone is insufficient to reduce macrophage content. 
Verhoeven et al. (2006) reported their interesting findings on macrophage content in carotid atherosclerotic plaques from a clinical study in 378 patients with or without statin treatment. They found that although serum cholesterol, low-density lipoprotein, C-reactive protein, and carotid atheromatous plaques were reduced in patients treated with statins, CD68-positive cells were increased in atherosclerotic lesions. Furthermore, they found that the expression of IL6, a major proinflammatory cytokine expressed by macrophages (Li et al. 2010), was lower in atherosclerotic plaques in patients treated with statins. This study suggests that statin treatment attenuates macrophage activation, but not the recruitment of macrophages. Indeed, we also found that insulin treatment inhibited diabetes-increased IL6 expression.

In conclusion, this study showed that insulin treatment in diabetic apoE $-/-$ mice led to a reduction in intimal lesions and rebalance between MMP9 and TIMP1 expression. This study also showed that insulin treatment inhibited proinflammatory cytokine IL6 expression in atherosclerotic lesions but did not decrease diabetes-increased macrophage content. These findings indicate that insulin treatment may reduce cardiovascular events by stabilizing advanced atherosclerosis.

\section{Declaration of interest}

The authors declare that there is no conflict of interest that could be perceived as prejudicing the impartiality of the research reported.

\section{Funding}

This study was supported by a Merit Review Grant from the Department of Veterans Affairs and NIH grant DE016353 (to Y H).

\section{References}

Aljada A, Ghanim H, Mohanty P, Hofmeyer D, Tripathy D \& Dandona P 2001 Hydrocortisone suppresses intranuclear activator-protein-1 (AP-1) binding activity in mononuclear cells and plasma matrix metalloproteinase 2 and 9 (MMP-2 and MMP-9). Journal of Clinical Endocrinology and Metabolism 86 5988-5991. (doi:10.1210/jc.86.12.5988)

Bai YL, Huang HC, Li JZ, Zhao YY \& Wang HY 2005 High glucose regulates the production of MMP-9 in podocyte through ERK1/2 signal pathway. Zhonghua Yi Xue Za Zhi 85 1451-1455.

Blankenberg S, Rupprecht HJ, Poirier O, Bickel C, Smieja M, Hafner G, Meyer J, Cambien F \& Tiret L 2003 Plasma concentrations and genetic variation of matrix metalloproteinase 9 and prognosis of patients with cardiovascular disease. Circulation 107 1579-1585. (doi:10.1161/01.CIR. $0000058700.41738 .12)$

Brocheriou I, Maouche S, Durand H, Braunersreuther V, Le Naour G, Gratchev A, Koskas F, Mach F, Kzhyshkowska J \& Ninio E 2011 Antagonistic regulation of macrophage phenotype by M-CSF and GM-CSF: implication in atherosclerosis. Atherosclerosis 214 316-324. (doi:10.1016/j.atherosclerosis.2010.11.023)

Candido R, Allen TJ, Lassila M, Cao Z, Thallas V, Cooper ME \& JandeleitDahm KA 2004 Irbesartan but not amlodipine suppresses diabetesassociated atherosclerosis. Circulation 109 1536-1542. (doi:10.1161/01. CIR.0000124061.78478.94)
Collins AR, Meehan WP, Kintscher U, Jackson S, Wakino S, Noh G, Palinski W, Hsueh WA \& Law RE 2001 Troglitazone inhibits formation of early atherosclerotic lesions in diabetic and nondiabetic low density lipoprotein receptor-deficient mice. Arteriosclerosis, Thrombosis, and Vascular Biology 21 365-371.

Falk E, Shah PK \& Fuster V 1995 Coronary plaque disruption. Circulation 92 657-671.

Galis ZS \& Khatri JJ 2002 Matrix metalloproteinases in vascular remodeling and atherogenesis: the good, the bad, and the ugly. Circulation Research 90 251-262.

Galis ZS, Sukhova GK, Lark MW \& Libby P 1994 Increased expression of matrix metalloproteinases and matrix degrading activity in vulnerable regions of human atherosclerotic plaques. Journal of Clinical Investigation 94 2493-2503. (doi:10.1172/JCI117619)

Gatti A, Maranghi M, Bacci S, Carallo C, Gnasso A, Mandosi E, Fallarino M, Morano S, Trischitta V \& Filetti S 2009 Poor glycemic control is an independent risk factor for low HDL cholesterol in patients with type 2 diabetes. Diabetes Care 32 1550-1552. (doi:10. 2337/dc09-0256)

Gray JL \& Shankar R 1995 Down regulation of CD11b and CD18 expression in atherosclerotic lesion-derived macrophages. American Surgeon 61 674-679 (discussion 679-680).

Gurjar MV, Sharma RV \& Bhalla RC 1999 eNOS gene transfer inhibits smooth muscle cell migration and MMP-2 and MMP-9 activity. Arteriosclerosis, Thrombosis, and Vascular Biology 19 2871-2877.

Hayek T, Hussein K, Aviram M, Coleman R, Keidar S, Pavoltzky E \& Kaplan M 2005 Macrophage foam-cell formation in streptozotocininduced diabetic mice: stimulatory effect of glucose. Atherosclerosis $\mathbf{1 8 3}$ 25-33. (doi:10.1016/j.atherosclerosis.2005.02.018)

Jarrett RJ 1981 Atherosclerosis, diabetes and obesity. Proceedings of the Nutrition Society 40 209-212. (doi:10.1079/PNS19810030)

Khan HA, Sobki SH \& Khan SA 2007 Association between glycaemic control and serum lipids profile in type 2 diabetic patients: HbA1c predicts dyslipidaemia. Clinical and Experimental Medicine 7 24-29. (doi:10.1007/ s10238-007-0121-3)

Lee SJ, Bae SS, Kim KH, Lee WS, Rhim BY, Hong KW \& Kim CD 2007 High glucose enhances MMP-2 production in adventitial fibroblasts via Akt1-dependent NF-kappaB pathway. FEBS Letters 581 4189-4194. (doi:10.1016/j.febslet.2007.07.058)

Lehr HA, Mankoff DA, Corwin D, Santeusanio G \& Gown AM 1997 Application of photoshop-based image analysis to quantification of hormone receptor expression in breast cancer. Journal of Histochemistry and Cytochemistry 45 1559-1565. (doi:10.1177/00221554970 4501112)

Li Y, Samuvel DJ, Sundararaj KP, Lopes-Virella MF \& Huang Y 2010 IL-6 and high glucose synergistically upregulate MMP-1 expression by U937 mononuclear phagocytes via ERK1/2 and JNK pathways and c-Jun. Journal of Cellular Biochemistry 110 248-259. (doi:10.1002/jcb.22532)

Libby P 1995 Molecular bases of the acute coronary syndromes. Circulation $\mathbf{9 1}$ 2844-2850.

Libby P \& Theroux P 2005 Pathophysiology of coronary artery disease. Circulation 111 3481-3488. (doi:10.1161/CIR CULATIONAHA.105. 537878)

Liu CP, Yeh JL, Wu BN, Chai CY, Chen IJ \& Lai WT 2011 KMUP-3 attenuates ventricular remodelling after myocardial infarction through eNOS enhancement and restoration of MMP-9/TIMP-1 balance. British Journal of Pharmacology 162 126-135. (doi:10.1111/j.1476-5381.2010. 01024.x)

Loftus IM, Naylor AR, Bell PR \& Thompson MM 2001 Plasma MMP-9 - a marker of carotid plaque instability. European Journal of Vascular and Endovascular Surgery 21 17-21. (doi:10.1053/ejvs.2000.1278)

Marx N, Froehlich J, Siam L, Ittner J, Wierse G, Schmidt A, Scharnagl H, Hombach V \& Koenig W 2003 Antidiabetic PPAR gamma-activator rosiglitazone reduces MMP-9 serum levels in type 2 diabetic patients with coronary artery disease. Arteriosclerosis, Thrombosis, and Vascular Biology 23 283-288. (doi:10.1161/01.ATV.0000054195.35121.5E) 
McKellar GE, McCarey DW, Sattar N \& McInnes IB 2009 Role for TNF in atherosclerosis? Lessons from autoimmune disease Nature Reviews. Cardiology 6 410-417. (doi:10.1038/nrcardio.2009.57)

Montaner J, Molina CA, Monasterio J, Abilleira S, Arenillas JF, Ribo M, Quintana M \& Alvarez-Sabin J 2003 Matrix metalloproteinase-9 pretreatment level predicts intracranial hemorrhagic complications after thrombolysis in human stroke. Circulation 107 598-603. (doi:10.1161/01. CIR.0000046451.38849.90)

Moreno PR, Murcia AM, Palacios IF, Leon MN, Bernardi VH, Fuster V \& Fallon JT 2000 Coronary composition and macrophage infiltration in atherectomy specimens from patients with diabetes mellitus. Circulation 102 2180-2184.

Nakashima Y, Plump AS, Raines EW, Breslow JL \& Ross R 1994 ApoEdeficient mice develop lesions of all phases of atherosclerosis throughout the arterial tree. Arteriosclerosis and Thrombosis 14 133-140.

Nareika A, Sundararaj KP, Im YB, Game BA, Lopes-Virella MF \& Huang Y 2009 High glucose and interferon gamma synergistically stimulate MMP-1 expression in U937 macrophages by increasing transcription factor STAT1 activity. Atherosclerosis 202 363-371. (doi:10.1016/j.atherosclerosis.2008. 05.043)

Nathan DM, Cleary PA, Backlund JY, Genuth SM, Lachin JM, Orchard TJ, Raskin P \& Zinman B 2005 Intensive diabetes treatment and cardiovascular disease in patients with type 1 diabetes. New England Journal of Medicine 353 2643-2653. (doi:10.1056/NEJMoa052187)

Park L, Raman KG, Lee KJ, Lu Y, Ferran LJ Jr, Chow WS, Stern D \& Schmidt AM 1998 Suppression of accelerated diabetic atherosclerosis by the soluble receptor for advanced glycation endproducts. Nature Medicine 4 1025-1031. (doi:10.1038/2012)

Richardson PD, Davies MJ \& Born GV 1989 Influence of plaque configuration and stress distribution on fissuring of coronary atherosclerotic plaques. Lancet 2 941-944. (doi:10.1016/S0140-6736(89)90953-7)

Soedamah-Muthu SS \& Stehouwer CD 2005 Cardiovascular disease morbidity and mortality in patients with type 1 diabetes mellitus: management strategies. Treatments in Endocrinology 4 75-86. (doi:10.2165/ 00024677-200504020-00002)
Sundstrom C \& Nilsson K 1976 Establishment and characterization of a human histiocytic lymphoma cell line (U-937). International Journal of Cancer 17 565-577. (doi:10.1002/ijc.2910170504)

Tang Y \& Li GD 2004 Chronic exposure to high glucose impairs bradykininstimulated nitric oxide production by interfering with the phospholipaseC-implicated signalling pathway in endothelial cells: evidence for the involvement of protein kinase C. Diabetologia 47 2093-2104. (doi:10.1007/ s00125-004-1589-y)

Tse J, Martin-McNaulty B, Halks-Miller M, Kauser K, DelVecchio V, Vergona R, Sullivan ME \& Rubanyi GM 1999 Accelerated atherosclerosis and premature calcified cartilaginous metaplasia in the aorta of diabetic male Apo E knockout mice can be prevented by chronic treatment with 17 beta-estradiol. Atherosclerosis 144 303-313. (doi:10.1016/S00219150(98)00325-6)

Verhoeven BA, Moll FL, Koekkoek JA, van der Wal AC, de Kleijn DP, de Vries JP, Verheijen JH, Velema E, Busser E, Schoneveld A et al. 2006 Statin treatment is not associated with consistent alterations in inflammatory status of carotid atherosclerotic plaques: a retrospective study in 378 patients undergoing carotid endarterectomy. Stroke 37 2054-2060. (doi:10.1161/ 01.STR.0000231685.82795.e5)

Yadav A, Saini V \& Arora S 2010 MCP-1: chemoattractant with a role beyond immunity: a review. Clinica Chimica Acta 411 1570-1579. (doi:10.1016/j. cca.2010.07.006)

Yakubenko VP, Belevych N, Mishchuk D, Schurin A, Lam SC \& Ugarova TP 2008 The role of integrin alpha D beta2 (CD11d/CD18) in monocyte/macrophage migration. Experimental Cell Research 314 2569-2578. (doi:10.1016/j.yexcr.2008.05.016)

\section{Received in final form 21 March 2011 \\ Accepted 8 April 2011 \\ Made available online as an Accepted \\ Preprint 8 April 2011}

